\title{
Detecção automática de arritmia cardíaca em procedimento de Hemodiálise usando Random Forests
}

\author{
Sergio Pinto Gomes Junior \\ PEE/COPPE \\ Universidade Federal do Rio de Janeiro \\ Rio de Janeiro, Brasil \\ sergio.junior@coppe.ufrj.br
}

\author{
João Baptista de Oliveira e Souza Filho \\ $P E E / C O P P E$ \\ Universidade Federal do Rio de Janeiro \\ Rio de Janeiro, Brasil \\ jbfilho@poli.ufrj.br
}

\author{
Felipe da Rocha Henriques \\ PPCIC, PPGIO \\ CEFET/RJ \\ Petrópolis, Brasil \\ felipe.henriques@cefet-rj.br
}

\author{
Michel Pompeu Tcheou \\ PEL \\ Universidade do Estado do Rio de Janeiro \\ Rio de Janeiro, Brasil \\ mtcheou@uerj.br
}

\begin{abstract}
Resumo-Na literatura médica, a arritmia é uma das principais complicações que os pacientes submetidos à hemodiálise podem enfrentar, com destaque para as contrações prematuras atriais e ventriculares. Devido a tais complicações, torna-se tipicamente necessário que profissionais de saúde examinem periodicamente esses pacientes. Nesta direção, este trabalho propôs a avaliação de três classificadores: uma rede neural e os modelos de K-Nearest Neighbors (KNN) e Random Forests, para a identificação automática destes dois tipos de arritmia em pacientes submetidos a hemodiálise, o qual possa contribuir para uma resposta mais rápida da equipe e um possível melhor manejo das intercorrências. Para tanto, não é considerada nenhuma técnica de extração de parâmetros dos sinais, sendo o classificador alimentado com trechos dos sinais centrados nos batimentos, onde cada batimento define uma amostra de avaliação. Através de simulações computacionais, utilizando de uma base de dados reais de Eletrocardiograma (ECG), verificou-se que o classifcador Random Forests obteve uma melhor relação entre custo computacional e desempenho, alcançando uma acurácia de 98,6\% e uma sensibilidade de $92,0 \%$.
\end{abstract}

palavras-chave-Hemodiálise, arritmia, classificador de batimentos, Random Forests, apoio a decisão clínica

\section{INTRODUÇÃo}

Os rins executam algumas funções muito importantes para o corpo humano, como retirar do corpo o excesso de sal e de líquidos, controlar a pressão arterial e ajudar o organismo a manter o equilíbrio de substâncias, tais como o sódio, o potássio, a ureia e a creatinina. Um indivíduo com insuficiência renal pode, dependendo do nível de severidade da doença, necessitar de um procedimento chamado hemodiálise, no qual uma máquina executa o papel do rim que está doente [1]. Esse procedimento dura normalmente quatro horas e deve ser repetido em média três vezes por semana [2].

A hemodiálise é cada vez mais segura; no entanto, de acordo com [3], apesar das inovações tecnológicas neste procedimento, em aproximadamente $30 \%$ das sessões, pode ocorrer algum tipo de intercorrência, tais como hipotensão, hipertensão, a síndrome do desequilíbrio da diálise, arritmias cardíacas e a hipoxemia, sendo a arritmia uma das mais observadas.

De acordo com a Sociedade Brasileira de Cardiologia, as arritmias cardíacas são provocadas por distúrbios nos estímulos elétricos a que o coração está submetido [4], caracterizandose pela ocorrência de batimentos anormais que alteram o ritmo cardíaco para mais acelerado (taquicardia); mais lento (bradicardia); ou irregular [2] [3]. Esse distúrbio pode ser identificado por meio da análise, por um profissional da área da saúde, de um exame chamado eletrocardiograma (ECG), que é um método não-invasivo, utilizado na prática clínica para se detectar ocorrências de comportamentos anormais da atividade elétrica cardíaca [5].

A interpretação dos sinais de um ECG é essencialmente uma tarefa de reconhecimento de padrões. Muitos trabalhos realizaram a extração de parâmetros específicos de tais sinais se utilizando de técnicas tais como a transformada Wavelet [6] [7] [8] e a análise de componentes principais [9], previamente a etapa de classificação dos batimentos. Entretanto, outros trabalhos procuraram não realizar tal extração, alimentando seus modelos diretamente com os trechos do sinal de ECG que caracterizam os batimentos [10] [11]. Relativamente aos métodos de aprendizado de máquina investigados na literatura, estes incluem máquinas de vetores de suporte [12] [13], árvores de decisão, Random Forests [14] [15] e redes neurais [16] [17] [8]. Além de considerar diferentes estratégias para a extração dos parâmetros dos sinais envolvidos e considerar diferentes escolhas para os classificadores, trabalhos anteriores propuseram a detecção de diferentes tipos de arritmia. Em [18], o estudo teve como foco a classificação de cinco tipos de 
batimentos mais comuns da base de dados MIT-BIH Arrhythmia Database [19]: o batimento normal, o bloqueio dos ramos esquerdo e direito do feixe de His, a contração ventricular prematura e o batimento compassado. Por sua vez em [20], as amostras foram divididas em duas classes: batimentos normais e anormais, onde a última era composta por batimentos com contração ventricular prematura, batimentos supraventriculares prematuros e uma fusão entre batimentos ventriculares e normais. Na referência [21], verificou-se ainda que as arritmias mais frequentes em pacientes submetidos à hemodiálise são as contrações atriais (CAP) e as ventriculares (CVP) prematuras, que ocorreram, respectivamente, em $40,30 \%$ e $59,70 \%$ dos pacientes acompanhados. Baseando-se nesta constatação, o presente trabalho concentra-se apenas na identificação destes dois tipos de arritmia.

Neste trabalho é proposto um sistema de detecção automática de arritmia capaz de auxiliar uma equipe de saúde na análise dos batimentos de um paciente submetido à hemodiálise e, também, reportar a equipe a ocorrência de um caso de arritmia, contribuindo, assim, para uma reposta rápida a essa modalidade de intercorrência sofrida pelo paciente.

Este trabalho está estruturado da seguinte forma: na Seção II, apresenta-se uma breve descrição dos sinais de ECG. Em seguida, na Seção III, o sistema proposto neste trabalho é apresentado, iniciando-se com uma visão geral da proposta, seguido por um detalhamento do tratamento dos dados utilizados para o treinamento dos classificadores, e finalizado com a análise dos experimentos obtidos com três técnicas de classificação. Por sua vez, na Seção IV, os resultados obtidos são discutidos, realizando-se uma comparação com outros trabalhos da literatura, enquanto na Seção V são contempladas as considerações finais deste trabalho.

\section{SINAIS DE ECG}

O exame de ECG tem como resultado um sinal variante no tempo que reflete o fluxo de corrente responsável pela contração e o relaxamento das fibras do coração. Este sinal é obtido por meio da diferença de potencial medida entre dois eletrodos posicionados no corpo de um indivíduo. Em um ciclo de um ECG normal podem ser observadas as despolarizações e repolarizações elétricas do átrio e do ventrículo, que ocorrem a cada batimento cardíaco [22]. Tais eventos estão associados aos picos e vales da forma de onda de um ECG típico, sendo rotulados como P, Q, R, S e T, conforme ilustrado na Figura 1. Assim, para um batimento normal, podem-se observar os seguintes elementos [22]:

- Onda P: representa uma região de baixa tensão, que reflete a despolarização atrial;

- Complexo QRS: corresponde ao registro causado pela corrente gerada na despolarização do ventrículo. Neste instante, ocorre também a repolarização do átrio, mas ela não é registrada no ECG, devido a grande amplitude desse complexo;

- Onda T: é representado pelo trecho onde ocorre a repolarização ventricular, no qual há uma preparação do coração para um novo ciclo.

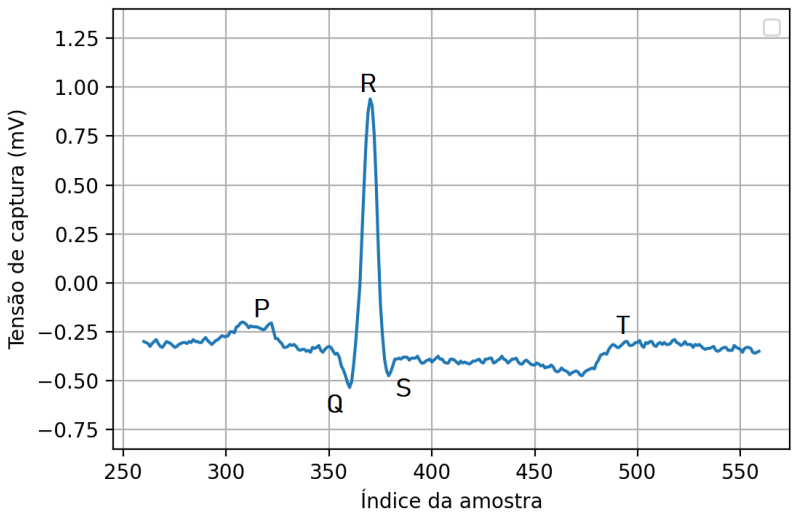

Figura 1. Seções do sinal de um ECG

Relativamente aos padrões observados em um ECG comumente empregados para a identificação de arritmias, os que se relacionam com os intervalos R-R são os mais utilizados. Este intervalo é definido tendo como início o pico de um complexo QRS e fim o pico similar, porém integrante do próximo complexo [23] [24]. Outros parâmetros temporais, tais como o intervalo entre duas ondas $\mathrm{P}$, a duração de $\mathrm{P}$, a duração do complexo QRS, o intervalo entre a onda $\mathrm{P}$ e o pico $\mathrm{R}$, a duração da onda $\mathrm{T}$ e o intervalo entre $\mathrm{Q}$ e $\mathrm{T}$ também podem ser utilizados. Além disso, características morfológicas da onda $\mathrm{P}$, do complexo QRS e da onda $\mathrm{T}$ também podem ser incluídas nessa análise [25].

\section{Sistema Proposto}

\section{A. Visão geral do sistema de detecção de arritmia}

O sistema proposto neste trabalho, conforme ilustrado na Figura 2, consiste de um equipamento de eletrocardiograma conectado a um dispositivo cujo papel é receber os dados do ECG, detectar e segmentar os batimentos, classificá-los e, no caso da ocorrência de uma arritmia, realizar o envio de um alerta para a equipe de saúde. Após receber uma fração representativa do sinal do ECG de um paciente sob monitoramento, cabe ao dispositivo dividir os sinais em janelas com dois segundos centradas nos picos $\mathrm{R}$, anteriormente detectados por meio do algoritmo proposto por Pan e Tompkins [26], largamente utilizado na literatura. Concluídas estas etapas, o sinal está pronto para ser classificado. Caso seja classificado como arritmia, o sistema emite um alerta específico.

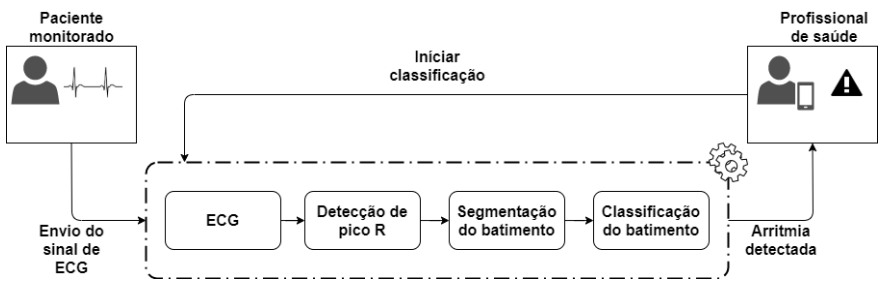

Figura 2. Sistema de detecção de arritmia 
Para realizar esta classificação, foi utilizado neste trabalho um modelo do tipo Random Forests, que avalia uma janela de 720 amostras temporais do sinal de ECG centrada no pico R, visando identificar a ocorrência de batimentos normais ou com arritmia. Cumpre destacar que as arritmias mais frequentes em pacientes submetidos à hemodiálise são as contrações atriais (CAP) e ventriculares (CVP) prematuras. A CAP corresponde a um batimento que ocorre antes do esperado, possuindo um complexo QRS mais estreito do que o normal. Uma exceção são os casos de CAP aberrante, para os quais o complexo QRS é mais largo do que o normal [28]. Por sua vez, as principais características da CVP são um amplo complexo QRS e a inexistência de uma onda $\mathrm{P}$ [29]. A título ilustrativo, as Figuras 3 e 4 apresentam trechos de sinais de ECG com sinais característicos da ocorrência desses dois tipos de arritmia.

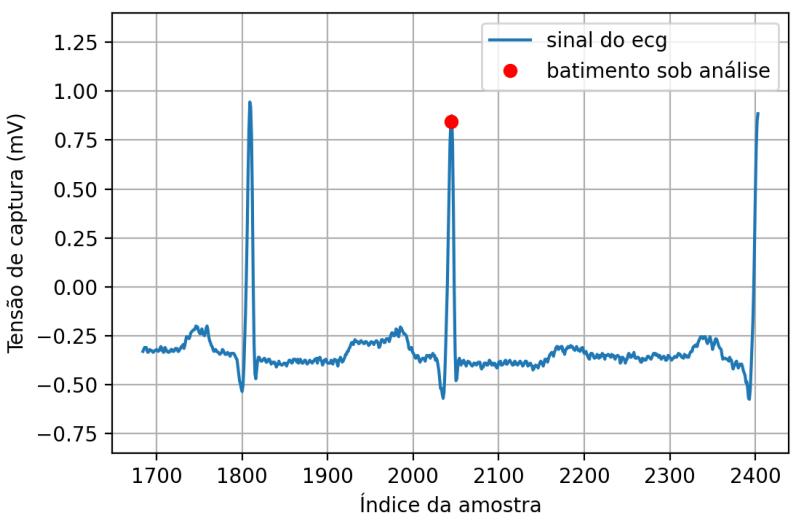

Figura 3. Arritmia do tipo contração atrial prematura (CAP)

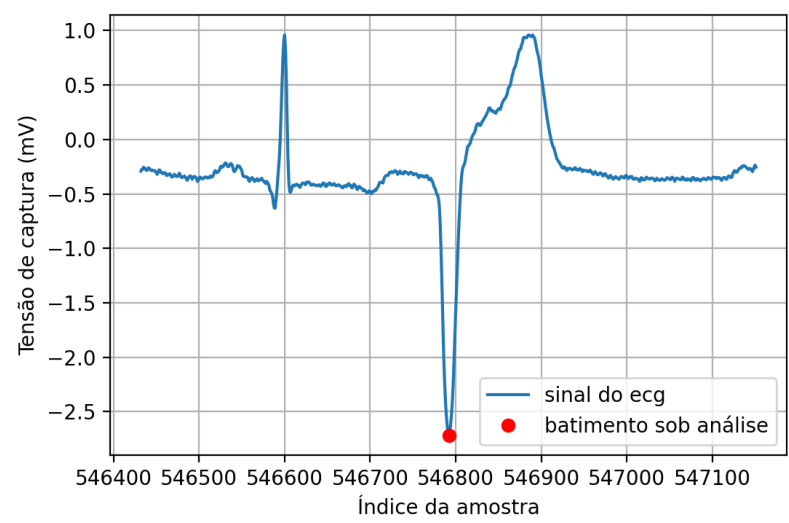

Figura 4. Arritmia do tipo contração ventricular prematura (CVP)

\section{B. Tratamento dos dados}

Os sinais utilizados neste trabalho foram extraídos da base de dados MIT-BIH Arrhythmia Database [19], disponível em PhisioNet [27]. Esta base é composta por 48 trechos, sendo cada um composto por meia hora de registro do ECG de um total de 47 pacientes ambulatoriais. Uma parte destes trechos foi extraída, de forma aleatória, de um conjunto de 4000 sinais de ECGs de 24 horas de duração. Já a outra parte dos sinais foi selecionada pelos autores da base, com o objetivo de incluir trechos com arritmias menos comuns, a fim de que a base de dados pudesse incluir uma grande diversidade de tipos de arritmia. Coube a dois médicos o papel de analisar os sinais dela integrantes de maneira independente, rotulando-os como normais ou característicos dos diversos tipos de arritmia. $\mathrm{O}$ tratamento das discordâncias foi dirimido por meio de uma reunião específica entre tais avaliadores. Tais rótulos foram, em sua maior parte, atribuídos à amostra correspondente ao pico $\mathrm{R}$ de cada batimento.

Tendo em vista os objetivos do presente trabalho, foi organizado um recorte da base de dados MIT-BIH Arrhythmia Database composto somente por sinais de ECG dos pacientes que apresentaram pelo menos um desses dois tipos de arritmias, correspondendo a 44 dos 48 sinais da base. Após essa etapa, estes sinais foram segmentados em trechos centralizados em cada pico $\mathrm{R}$ do sinal de ECG, que representa um batimento. Dado que a CAP e a CVP são contrações que ocorrem de forma prematura, tomou-se o cuidado de incluir nesses segmentos o momento anterior e o posterior a cada batimento para uma correta identificação de um caso de arritmia. Para realizar o dimensionamento ideal desses segmentos, foram realizados experimentos de validação cruzada com o auxílio do classificador Random Forests utilizado neste trabalho, considerando janelas de amostras de 2 a 8 segundos de duração. Entretanto, como não foi observada nenhuma diferença significativa entre os resultados dos experimentos, optou-se por uma janela mais curta, com duração de 2 segundos, de forma a reduzir a dimensão de entrada do classificador, resultando em janelas de 720 amostras, das quais 359 precedem o pico $R$ e 360 o sucedem. A Figura 5 apresenta um desses segmentos.

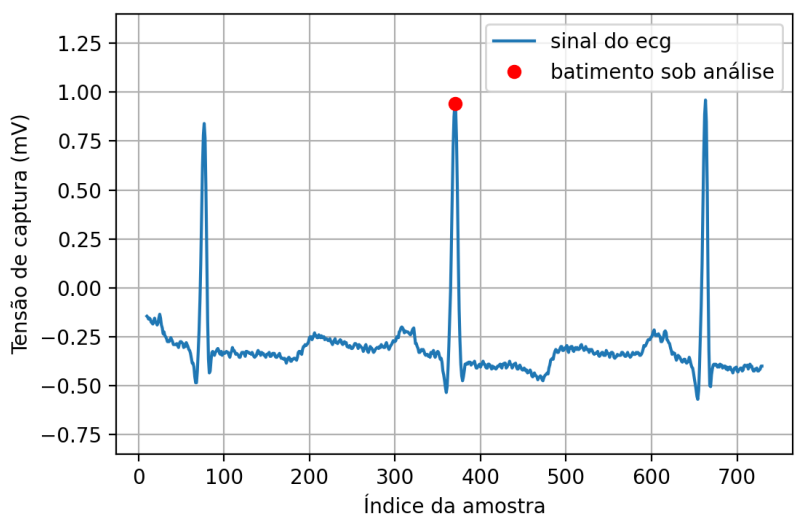

Figura 5. Um exemplo de batimento normal

Após essa segmentação, foram mantidos apenas os trechos contendo os batimentos normais e as arritmias CAP e CVP. Assim, o conjunto de dados utilizado neste trabalho foi separado em duas classes: uma de Batimentos Normais 
e outra de batimentos com Arritmia, distribuídos conforme apresentado na Tabela I. As classes foram definidas desta forma, pois considerou-se que, independentemente dos dois tipos de arritmia detectados, faz-se necessário acionar a equipe de saúde.

Tabela I

QUANTITATIVO DAS JANELAS DE OBSERVAÇÃO PARA CADA CLASSE DE INTERESSE

\begin{tabular}{ll}
\hline Classe & Janelas \\
\hline Batimento normal & 55820 \\
Arritmia (CAP + CVP) & 7979 \\
\hline
\end{tabular}

Este conjunto de dados foi então dividido aleatoriamente em três grupos: treino, validação e teste, onde cada grupo contemplou $56 \%, 14 \%$ e $30 \%$ dos dados, respectivamente. Adicionalmente, foi realizada a normalização das variáveis constituintes das janelas para que as mesmas possuíssem média zero e desvio padrão igual a um.

\section{Classificador}

Após a definição do conjunto de dados, com o objetivo de determinar um classificador para a aplicação proposta neste trabalho, três modelos foram treinados para classificar os batimentos cardíacos nas duas categorias supracitadas (normal e arritimia).

O primeiro modelo avaliado foi uma rede neural artificial Multilayer Perceptron que possuiu uma única camada escondida com neurônios cujas funções de ativação são do tipo tangente hiperbólica, enquanto o neurônio da camada de saída possui função de ativação do tipo sigmoide, visto que tal rede realiza uma classificação binária. Além disso, o método de otimização escolhido foi o Gradiente Descendente Estocástico (Stochastic Gradient Descent - SGD) [30], adotando-se como função custo a entropia cruzada, dada por

$$
E=-\sum_{i=1}^{n}\left[\left(1-d_{i}\right) \log _{2}\left(1-a_{i}\right)+d_{i} \log _{2}\left(a_{i}\right)\right],
$$

onde $a_{i}$ é a saída da rede para um evento de entrada $\mathbf{x}_{i}$, enquanto $d_{i}$ representa a saída desejada correspondente.

Para se encontrar valores ideais relativamente ao número de neurônios da camada escondida e a taxa de aprendizagem a ser utilizada no SGD, foi realizada uma busca em grade. Segundo este método, os ensaios consideraram uma quantidade de neurônios na camada escondida na faixa de 5 a 15 , em intervalos de 5, e taxas de aprendizagem no conjunto: $\{0,001 ; 0,01$ e 0,1$\}$. Para cada configuração, as redes foram treinadas com o auxílio do método de validação cruzada, cujo objetivo é reduzir o overfitting, onde a divisão dos dados respeitou o balanceamento das classes. A etapa de treinamento e validação foi repetida cinco vezes, considerando-se diferentes inicializações para os parâmetros das redes. A Tabela II resume os valores de acurácia média e de desvio padrão, entre parênteses, obtidos pela busca em grade. A partir destes resultados, foi definido que a rede neural adotaria quinze neurônios na camada escondida e uma taxa de aprendizagem igual a 0,01 .

Tabela II

RESULTADOS DE ACURÁCIA MÉDIA E DE DESVIO PADRÃO OBTIDOS NA BUSCA EM GRADE PARA A REDE NEURAL

\begin{tabular}{|c|c|c|c|c|}
\hline & \multicolumn{3}{|c|}{ Taxa de aprendizagem } \\
\hline & & 0,001 & 0,01 & 0,1 \\
\hline \multirow{4}{*}{$\begin{array}{c}\text { Número } \\
\text { de neurônios }\end{array}$} & & $97,43 \%$ & $97,72 \%$ & $97,53 \%$ \\
\hline & 5 & $( \pm 2,75 \%)$ & $( \pm 2,27 \%)$ & $( \pm 0,20 \%)$ \\
\hline & 10 & $\begin{array}{c}97,81 \% \\
\end{array}$ & $\begin{array}{c}98,23 \% \\
\end{array}$ & $\begin{array}{c}97,60 \% \\
(+0.12 \%\end{array}$ \\
\hline & 15 & $\begin{array}{c}98,15 \% \\
(+0.22 \%)\end{array}$ & $\begin{array}{c}\mathbf{9 8 , 5 0 \%} \\
(+0,12 \%)\end{array}$ & $\begin{array}{c}97,95 \% \\
(+0.24 \%)\end{array}$ \\
\hline
\end{tabular}

O segundo modelo avaliado foi o Random Forests, considerando-se também uma busca em grade para o ajuste da profundidade e do número de árvores. Os resultados desta busca são sintetizados na Tabela III, tendo levado a definição de uma arquitetura com 80 árvores de profundidade igual a 30 .

Tabela III

RESULTADOS DE ACURÁCIA MÉDIA E DE DESVIO PADRÃo OBTIDOS NA BUSCA EM GRADE PARA O Random Forests

\begin{tabular}{lcccc}
\cline { 3 - 4 } & & \multicolumn{3}{c}{ Árvores } \\
\cline { 3 - 5 } & \multirow{2}{*}{ Profundidade } & $98,40 \%$ & 90 & 100 \\
\hline & \multirow{2}{*}{20} & $( \pm 0,11 \%)$ & $( \pm 0,39 \%$ & $98,42 \%$ \\
& \multirow{2}{*}{25} & $( \pm 0,54 \%$ & $98,59 \%)$ & $( \pm 0,08 \%)$ \\
& & $( \pm 0,15 \%)$ & $( \pm 0,10 \%)$ & $( \pm 0,15 \%$ \\
& \multirow{2}{*}{30} & $\mathbf{9 8 , 6 6 \%}$ & $98,64 \%$ & $98,64 \%$ \\
& $( \pm 0,15 \%)$ & $( \pm 0,11 \%)$ & $( \pm 0,11 \%)$ \\
\hline
\end{tabular}

O último experimento considerou um classificador baseado no algoritmo K-Nearest Neighbors (KNN), cujos hiperparâmetros envolvidos na busca em grade foram o número de vizinhos e o modelo de atribuição de pesos aos pontos utilizados para a predição, isto é, se tais pesos seriam uniformes ou corresponderiam ao inverso das distâncias aos dado de treino. Os resultados correspondentes são apresentados na Tabela IV, tendo motivado a escolha de quatro vizinhos e pesos associados ao inverso das distâncias.

Tabela IV

RESULTADOS DE ACURÁCIA MÉDIA E DE DESVIO PADRÃo OBTIDOS NA BUSCA EM GRADE ASSOCIADA A TÉCNICA KNN

\begin{tabular}{cccc}
\cline { 3 - 3 } & \multicolumn{2}{c}{ Pesos } \\
\cline { 3 - 4 } & 4 & Uniforme & Distância \\
\hline \multirow{2}{*}{ Vizinhos } & 5 & $98,13 \%( \pm 0,07 \%)$ & $\mathbf{9 8 , 3 5 \%}( \pm 0,09 \%)$ \\
& 6 & $97,98 \%( \pm 0,11 \%)$ & $98,16 \%( \pm 0,11 \%)$ \\
& &
\end{tabular}

Após terem sido definidos seus hiperparâmetros, os três classificadores foram avaliados com o conjunto de teste. A Tabela $\mathrm{V}$ resume os valores de algumas métricas de avaliação de desempenho típicas para os três métodos.

Ainda que os resultados tenham sido muito próximos, o modelo Random Forests atingiu os melhores resultados para todas as métricas. Ademais, se comparado a rede neural, a qual apresenta os mesmos valores de sensibilidade e recall, este 
Tabela V

RESUltados DE ALGUMAS MÉTRICAS DE DESEMPENHO PARA OS TRÊS CLASSIFICADORES AVALIADOS

\begin{tabular}{lccc}
\cline { 2 - 4 } \multicolumn{1}{c}{ Métrica } & \multicolumn{3}{c}{ Rede } \\
& Neural & $\begin{array}{c}\text { Random } \\
\text { Forests }\end{array}$ & \multirow{2}{*}{ KNN } \\
\hline Acurácia & $98,7 \%$ & $\mathbf{9 8 , 8 \%}$ & $98,6 \%$ \\
Sensibilidade & $\mathbf{9 2 , 0 \%}$ & $\mathbf{9 2 , 0 \%}$ & $91,0 \%$ \\
Especificade & $99,6 \%$ & $\mathbf{9 9 , 8 \%}$ & $99,7 \%$ \\
Recall & $\mathbf{9 2 , 0 \%}$ & $\mathbf{9 2 , 0 \%}$ & $91,0 \%$ \\
Precisão & $97,1 \%$ & $\mathbf{9 8 , 3 \%}$ & $97,7 \%$ \\
F1 & $94,5 \%$ & $\mathbf{9 5 , 0 \%}$ & $94,2 \%$ \\
\hline
\end{tabular}

possui uma complexidade e custo computacional em fase de operação significativamente inferiores. A título de ilustração, o modelo Random Forests inclui 80 árvores de 30 níveis, levando a um total de 2400 comparações e a realização de uma média de 80 valores. Por sua vez, a rede neural demanda, só em sua primeira camada, 15 produtos internos entre vetores de dimensão 720 (entrada x pesos dos neurônios), resultando na ordem de 10800 multiplicações e somas. Deste modo, a solução Random Forests é mais atrativa computacionalmente para ampla gama de plataformas, em especial considerando uma implementação IoT (Internet of Things) do sistema proposto.

Além da sua menor complexidade e bom desempenho, o algortimo Random Forests possui uma vantagem adicional sobre os demais: permitir uma fácil identificação de quais são as amostras mais importantes para a detecção de arritmias. Para isso, foi realizada uma análise da importância de cada atributo através do indicador feature importance, que se baseia no cálculo da média do acúmulo da diminuição de impurezas dentro de cada árvore do comitê ilustrada na Figura 6. Como se pode observar, as amostras mais próximas a posição do pico R, isto é, na posição central (amostra 360), são as mais importantes para a predição. Cabe destacar que este resultado é consistente com a análise visual do sinal de ECG de uma CVP, onde pode ser verificada uma aparência específica para os complexos QRS, diferentemente de um batimento normal, previamente ilustrado na Figura 4. Por sua vez, a CAP pode ser identificada pela ocorrência antecipada do batimento e pela duração mais reduzida do complexo QRS [28], corroborando com o achado relativo à importância das amostras próximas ao pico $\mathrm{R}$ para a detecção de arritmia.

\section{Discussẽo}

Muitos métodos para a detecção automática e a classificação de diversos tipos de arritmias tem sido propostos na Literatura. A Tabela VI resume os valores de acurácia, os classificadores e os conjuntos de dados adotados por métodos estado-daarte quanto à classificação de batimento em sinais de ECG, considerando a base de dados MIT-BIH Arrhythmia Database. Desta tabela, pode ser observado que a maioria dos trabalhos considera diferentes tipos de batimentos no processo de classificação, excetuando-se a [9], que, como este trabalho, propôs um modelo para detectar uma única classe de batimentos anormais composta por contrações ventriculares e atriais

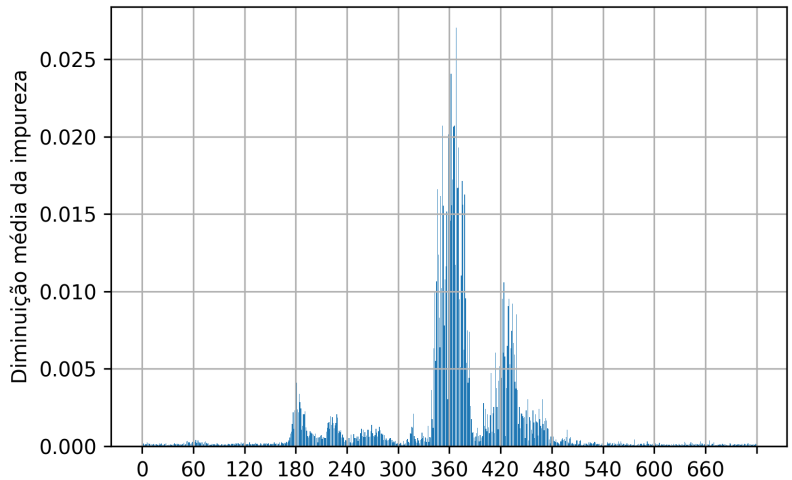

Figura 6. Importância dos parâmetros utilizando a diminuição média das impurezas

prematuras. Tendo-se em vista as diferenças entre as categorias de classificação e as partições da base de dados consideradas por estes trabalhos, pode-se considerar os resultados aqui obtidos como bastante satisfatórios. Cumpre-se destacar que o modelo proposto é o mais simples e de menor complexidade computacional em fase de operação dentre todas as alternativas estado-da-arte da Literatura, sendo apenas superado por um modelo de aprendizado profundo que possui uma complexidade significativamente maior.

Cabe ainda observar que o desbalanço entre as classes normal e arritmia é da ordem de 7 para 1 . A exploração de estratégias para a mitigação de possíveis efeitos deste desbalanço pode ainda levar a uma melhoria do desempenho, ação reservada para trabalhos futuros.

\section{CONClusão E Futuras diREÇões}

O presente trabalho discutiu a construção de um sistema sistema de alerta da ocorrência de arritmias em pacientes sob hemodiálise. A abordagem proposta por este trabalho, que considera trechos dos sinais de ECG centrados em batimentos para a definição de janelas simétricas de amostras que alimentam um classificador do tipo Random Forests, apresentou resultados comparáveis à outras abordagens na literatura, porém resultou em um modelo significativamente mais simples, logo com complexidade computacional expressivamente inferior.

Relativamente a modelos alternativos de classificação, a técnica de Random Forests apresentou resultados ligeiramente superiores para diferentes indicadores de desempenho.

Adicionalmente, por meio de uma análise da importância dos atributos, foi possível verificar que as amostras do complexo QRS foram identificadas como as mais importantes para a classificação, o que é compatível com a análise visual de um ECG por um profissional especialista que tipicamente considera o formato desse complexo para a identificação de arritmia.

Por fim, acredita-se que o desbalanceamento entre as classes possa ter influído na sensibilidade do modelo proposto. Nesta direção, em um trabalho futuro, espera-se avaliar técnicas de 
Tabela VI

MÉTOdos ESTADO-DA-ARTE PARA A CLASSIFICAÇ̃̃o DE BATIMENTOS EM ECG

\begin{tabular}{llll}
\hline \multicolumn{1}{c}{ Trabalho } & \multicolumn{1}{c}{ Classes de batimentos } & Classificador & Acurácia \\
\hline Dong et al. [18] & $\begin{array}{l}\text { Normal, bloqueio dos ramos esquerdo e direito do feixe de His, } \\
\text { contracão ventricular prematura e batimento compassado }\end{array}$ & Rede Neural RBF & $97,8 \%$ \\
\hline Inan et al. [31] & $\begin{array}{l}\text { Normal, contração ventricular prematura e outros } \\
\text { (bloqueio dos ramos esquerdo e direito do feixe de His, } \\
\text { batimento compassado e flutter atrial) }\end{array}$ & Rede Neural MLP & 95,2\% \\
\hline Martis et al. [9] & $\begin{array}{l}\text { Normal e anormal (contracão ventricular prematura e } \\
\text { contracão atrial prematura) }\end{array}$ & Máquina de Vetores de Suporte & $98,4 \%$ \\
\hline Sannino e De Pietro [20] & $\begin{array}{l}\text { normal e anormal (contração ventricular prematura, } \\
\text { batimentos supraventriculares prematuros e } \\
\text { fusão entre batimentos ventriculares e normais) }\end{array}$ & Reural Profunda & $99,5 \%$ \\
\hline Este trabalho & $\begin{array}{l}\text { Normal e anormal (contração ventricular prematura e } \\
\text { contração atrial prematura) }\end{array}$ & Random Forests & $98,8 \%$ \\
\hline
\end{tabular}

data augmentation, realizando-se, por exemplo, a adição de dados sintéticos a classe de arritmia [32], a fim de se buscar um aumento no valor da sensibilidade.

\section{REFERÊNCIAS}

[1] Brasil. "Diretrizes clínicas para o cuidado ao paciente com doença renal crônica-DRC no Sistema Único de Saúde." Secretaria de Atenção à Saúde 1 (2014): 1-37.

[2] F. de Souza Terra, et al. "As principais complicações apresentadas pelos pacientes renais crônicos durante as sessões de hemodiálise." Revista da Sociedade Brasileira de 8.3 (2010): 87

[3] M. C. M. de Castro. "Atualização em diálise: complicações agudas em hemodiálise." J Bras Nefrol 23.2 (2001): 108-13.

[4] J. C. Nicolau, et al. "Diretriz de interpretação de eletrocardiograma de repouso." Arquivos Brasileiros de Cardiologia 80 (2003): 1-18.

[5] C. J. Grupi, F. S. de Brito, A. H. Uchida. "Eletrocardiograma de Longa Duraçao: o Sistema Holter-Parte II." Journal of Carcdiac Arrhythmias 12.3 (1999): 134-146.

[6] E. S. Jayachandran, "Analysis of myocardial infarction using discrete wavelet transform." Journal of medical systems 34.6 (2010): 985-992.

[7] J. A. Gutiérrez-Gnecchi, et al. "DSP-based arrhythmia classification using wavelet transform and probabilistic neural network." Biomedical Signal Processing and Control 32 (2017): 44-56.

[8] N. K. Dewangan, S. P. Shukla. "ECG arrhythmia classification using discrete wavelet transform and artificial neural network." 2016 IEEE International Conference on Recent Trends in Electronics, Information and Communication Technology (RTEICT). IEEE, 2016.

[9] R. J. Martis, et al. "Application of higher order cumulants to ECG signals for the cardiac health diagnosis." 2011 Annual International Conference of the IEEE Engineering in Medicine and Biology Society. IEEE, 2011.

[10] Y. Ozbay, K. Bekir. "A recognition of ECG arrhytihemias using artificial neural networks." 2001 Conference Proceedings of the 23rd Annual International Conference of the IEEE Engineering in Medicine and Biology Society. Vol. 2. IEEE, 2001.

[11] S. S. Xu, M. W. Mak, C. C. Cheung. "Towards end-to-end ECG classification with raw signal extraction and deep neural networks." IEEE journal of biomedical and health informatics 23.4 (2018): 1574-1584.

[12] S. Faziludeen, P. V. Sabiq. "ECG beat classification using wavelets and SVM." 2013 IEEE Conference on Information and Communication Technologies. IEEE, 2013.

[13] N. Kohli, N. K. Verma, A. Roy. "SVM based methods for arrhythmia classification in ECG." 2010 international conference on computer and communication technology (ICCCT). IEEE, 2010.

[14] G. Pan, et al. "Arrhythmia classification based on wavelet transformation and random forests." Multimedia Tools and Applications 77.17 (2018): 21905-21922.

[15] N. Emanet. "ECG beat classification by using discrete wavelet transform and Random Forest algorithm." 2009 Fifth International Conference on Soft Computing, Computing with Words and Perceptions in System Analysis, Decision and Control. IEEE, 2009.

[16] J. S. Wang, et al. "ECG arrhythmia classification using a probabilistic neural network with a feature reduction method." Neurocomputing 116 (2013): $38-45$
[17] M. K. Sarkaleh, S. Asadollah. "Classification of ECG arrhythmias using discrete wavelet transform and neural networks." International Journal of Computer Science, Engineering and Applications 2.1 (2012): 1.

[18] X. Dong, C. Wang, W. Si. "ECG beat classification via deterministic learning." Neurocomputing 240 (2017): 1-12.

[19] G. B. Moody, R. G. Mark. "The impact of the MIT-BIH arrhythmia database." IEEE Engineering in Medicine and Biology Magazine 20.3 (2001): 45-50

[20] G. Sannino, G. De Pietro. "A deep learning approach for ECG-based heartbeat classification for arrhythmia detection." Future Generation Computer Systems 86 (2018): 446-455.

[21] N. Mahmood, et al. "Patterns of Cardiac Arrhythmia in Hemodialysis Patients." Anwer Khan Modern Medical College Journal 7.1 (2016): 28-33.

[22] P. E. McSharry, et al. "A dynamical model for generating synthetic electrocardiogram signals." IEEE transactions on biomedical engineering 50.3 (2003): 289-294.

[23] M. Llamedo, J. P. Martínez. "Heartbeat classification using feature selection driven by database generalization criteria." IEEE Transactions on Biomedical Engineering 58.3 (2010): 616-625.

[24] P. de Chazal, M. O'Dwyer, R. B. Reilly. "Automatic classification of heartbeats using ECG morphology and heartbeat interval features." IEEE transactions on biomedical engineering 51.7 (2004): 1196-1206.

[25] J. Wiens, J. Guttag. "Active learning applied to patient-adaptive heartbeat classification." Advances in neural information processing systems 23 (2010): 2442-2450.

[26] J. Pan, W. J. Tompkins. "A real-time QRS detection algorithm." IEEE transactions on biomedical engineering 3 (1985): 230-236.

[27] A. L. Goldberger, et al. "PhysioBank, PhysioToolkit, and PhysioNet: components of a new research resource for complex physiologic signals." circulation 101.23 (2000): e215-e220.

[28] D. Conen, et al. "Premature atrial contractions in the general population: frequency and risk factors." Circulation 126.19 (2012): 2302-2308.

[29] X. Liu, et al. "Automatic diagnosis of premature ventricular contraction based on Lyapunov exponents and LVQ neural network." Computer methods and programs in biomedicine 122.1 (2015): 47-55.

[30] S. Haykin. "Neural networks: a comprehensive foundation". Prentice hall, 1999.

[31] O. T. Inan, L. Giovangrandi, G. T. Kovacs. "Robust neural-networkbased classification of premature ventricular contractions using wavelet transform and timing interval features." IEEE transactions on Biomedical Engineering 53.12 (2006): 2507-2515.

[32] P. Wang, et al. "ECG arrhythmias detection using auxiliary classifier generative adversarial network and residual network." IEEE Access 7 (2019): 100910-100922. 